\title{
The characteristics and risk factors of central compartment lymph node metastasis in cNO papillary thyroid carcinoma coexistent with Hashimoto's thyroiditis
}

\author{
Kai Jin ${ }^{1,2 \#}$, Liang $\mathrm{Li}^{1,3 \#}$, Yahang $\mathrm{Liu}^{2}$, Xudong Wang ${ }^{1}$ \\ ${ }^{1}$ Department of Maxillofacial \& E.N.T Oncology, Tianjin Medical University Cancer Institute and Hospital, National Clinical Research Center for \\ Cancer, Tianjin's Clinical Research Center for Cancer, Key Laboratory of Cancer Prevention and Therapy, Tianjin, China; ${ }^{2}$ Department of Thyroid \\ Neoplasms Surgery, Inner Mongolia People's Hospital, Hohhot, China; ${ }^{3}$ Department of Otolaryngology, Tianjin Children's Hospital, Tianjin \\ University Children's Hospital, Tianjin, China \\ Contributions: (I) Conception and design: K Jin; (II) Administrative support: X Wang; (III) Provision of study materials or patients: All authors; (IV) \\ Collection and assembly of data: K Jin, L Li; (V) Data analysis and interpretation: K Jin, L Li; (VI) Manuscript writing: All authors; (VII) Final \\ approval of manuscript: All authors. \\ "These authors contributed equally to this work. \\ Correspondence to: Xudong Wang. Department of Maxillofacial \& E.N.T Oncology, Tianjin Medical University Cancer Institute and Hospital, \\ National Clinical Research Center for Cancer, Tianjin's Clinical Research Center for Cancer, Key Laboratory of Cancer Prevention and Therapy, \\ Tianjin, China. Email: wxd.1133@163.com.
}

\begin{abstract}
Background Currently, there are few studies on the characteristics of lymph node metastasis in the central region in patients with preoperative negative lymph node (cN0) papillary thyroid carcinoma (PTC) coexistent with Hashimoto's thyroiditis (HT). There is still a significant controversy on whether to perform prophylactic central compartment lymph node dissection for T1/T2 cN0 PTC. Therefore, we aimed to investigate the characteristics and risk factors of central compartment lymph node metastasis in cN0 PTC (T1 or T2 stage) coexists with HT.
\end{abstract}

Methods: From Jun. 2015 to Apr. 2019, the clinicopathological data of 354 patients with stage T1/ T2 cN0 PTC admitted to the thyroid tumor surgery of Inner Mongolia People's Hospital were analyzed retrospectively. All patients underwent central compartment lymph node dissection. According to the results of the postoperative pathological examination, the patients were divided into two groups: PTC group ( $\mathrm{n}=236$ ) and PTC coexistent with the HT group ( $\mathrm{n}=118)$.

Results: The proportion of PTC patients with HT was 33.33\% (118/354) in T1/T2 cN0 PTC patients; most of them were women. The levels of serum thyrotropin, antithyroglobulin antibody, and thyroid peroxidase antibody in PTC coexistent with HT group are higher than those in the PTC group $(\mathrm{P}<0.05)$. The number of lymphadenectomies in PTC coexistent with HT group was more than that in PTC alone group $(\mathrm{P}<0.05)$. Univariate analysis showed that antithyroglobulin antibody positive, tumor diameter $>1 \mathrm{~cm}$, and multifocal cancer in T1/T2 stage cN0 PTC coexistent with HT group were all correlated with lymph node metastasis in the central region $(\mathrm{P}<0.05)$. Logistic regression analysis showed that tumor diameter $>1 \mathrm{~cm}$, and multifocal cancer were the risk factors of central compartment lymph node metastasis in patients with T1/T2 stage cN0 PTC coexistent with HT $(\mathrm{P}<0.05)$.

Conclusions: HT is not a relevant factor of central lymph node metastasis in T1/T2 cN0 PTC; regardless of the presence or absence of HT, tumor diameter $>1 \mathrm{~cm}$ and multifocal cancer are risk factors for central lymph node metastasis in patients with T1/T2 cN0 PTC. Therefore, preventive lymph node dissection in the central region should be conducted actively during the operation.

Keywords: Papillary thyroid carcinoma (PTC); Hashimoto's thyroiditis (HT); central compartment lymph node 
Submitted Aug 11, 2020. Accepted for publication Oct 29, 2020.

doi: 10.21037 /gs-20-699

View this article at: http://dx.doi.org/10.21037/gs-20-699

\section{Introduction}

Thyroid cancer has become the fastest-growing malignant tumor in the world, with an annual growth rate of 4.5-6.6\%, and showing a rising trend year by year. The papillary thyroid carcinoma (PTC) accounts for $80-90 \%$ of all new thyroid cancer cases (1-3). Hashimoto's thyroiditis (HT) is the most common thyroid inflammatory disease, with an incidence of $0.3 \%$ to $5.1 \%$, accounting for $2 \%$ of the total population $(4,5)$. Since Lindsay et al. (6) proposed the correlation between HT and thyroid cancer in 1952, many studies have focused on the link between the two diseases. Some studies have shown PTC combined with $\mathrm{HT}$ is associated with lower central lymph node metastasis rate, lower invasive, and recurrence (7). However, the above relationship has not been confirmed in other studies, and its pathogenesis and long-term prognosis remain uncertain (8). Current studies have shown that lymph node metastatic carcinomas of thyroid origin are first distributed in the affected side area central compartment (level VI), followed by levels III, IV, and II, finally levels VII, I and V (9). When PTC coexistent with HT, the pathology can show that a large number of lymphocytes in the cancer tissue and adjacent to the cancer tissue are infiltrated and densely packed, or showed focal infiltration in the cancer parenchyma. At present, there are few studies on the characteristics of lymph node metastasis in the central region in patients with preoperative negative lymph node (cN0) PTC coexistent with HT. There is still a significant controversy on whether to perform prophylactic central compartment lymph node dissection (prophylactic central compartment lymph node dissection, PCCND) for T1/ T2 cN0 PTC. The 2015 American Thyroid Association guidelines recommend PCCND is not required for noninvasive T1/T2 cN0 PTC (10). The 2014 version of the British Thyroid Association guidelines recommended before PCCND is performed, individualized analysis of the relevant risk factors in $\mathrm{cN} 0$ PTC patients is required (11). The purpose of this study was to investigate the characteristics and risk factors of central compartment lymph node metastasis in patients with $\mathrm{T} 1 / \mathrm{T} 2 \mathrm{cN} 0$ PTC, coexistent with HT, to provide a reference for individualized clinical treatment. We present the following article in accordance with the STROBE reporting checklist (available at http://dx.doi.org/10.21037/gs-20-699).

\section{Methods}

\section{General information}

Retrospective analysis was performed on the complete case data of 354 patients with T1/T2 Stage cN0 PTC admitted to the Department of Thyroid Neoplasms Surgery, Inner Mongolia People's Hospital from June 2015 to April 2019, including 55 males and 299 females, with a male-tofemale ratio of 1:5.44. They were 21 to 75 years old, with an average age of $47.83 \pm 9.40$ years, of which 272 cases were younger than 55 years old, and 82 cases were older than 55 years old. According to postoperative pathological examination results, the patients were divided into the PTC group $(n=236)$ and PTC coexistent with the HT group $(\mathrm{n}=118)$. The study was reviewed and approved by the Ethics Committee of Clinical Investigation in the Inner Mongolia People's hospital. All procedures performed in this study involving human participants were in accordance with the Declaration of Helsinki (as revised in 2013). Individual consent for this retrospective analysis was waived.

\section{Inclusion and exclusion criteria}

Inclusion criteria: (I) preoperative diagnosis of T1/ T2 stage cN0 PTC adopts the AJCC 8th edition of the TNM staging standard for thyroid cancer (12). Central compartment lymph node dissection was performed during the operation, and histopathological examination confirmed PTC after the operation; (II) no earlier history of thyroid surgery; (III) unilateral or bilateral central lymph node dissection was performed intraoperatively; (IV) no levothyroxine or anti-thyroid drugs were taken one month before surgery; (V) have complete clinical pathological data. Exclusion criteria: (I) complicated with other autoimmune diseases; (II) complicated with other pathological types of thyroid malignancies; (III) secondary malignant tumors of the thyroid that originate from other sites; (IV) no history of head and neck radiotherapy and family history of malignant thyroid tumors. From the results of postoperative 
pathological examinations, patients were divided into PTC coexistent with HT group and pure PTC group. All samples were paraffin sectioned, and HE was stained and diagnosed by two experienced pathologists.

\section{Study methods}

We recorded the data of two groups of patients and noted gender, age, tumor diameter (multifocal carcinoma records the largest diameter), and number of cancers. Record the serum-free thyroxine concentration (FT4), thyroid-stimulating hormone concentration (TSH), antithyroglobulin antibody concentration (anti-TGAb), and thyroid peroxidase antibody concentration (TPO-Ab) of the two groups of patients before surgery. The proportion of lymph node metastasis in the central compartment region, numbers of lymph node dissection, numbers of lymph node metastasis in the central compartment region, and the TNM stage in the two groups are recorded. Compare the differences in clinical characteristics between the two groups and analyze the risk factors that affect lymph node metastasis in the central compartment region.

\section{Statistical analysis}

SPSS 20.0 statistical software was used for data analysis. Measurement data of normal distribution were expressed as mean \pm standard deviation $(\bar{x} \pm s)$, and an independent sample $t$-test was used for comparison between the two groups. Enumeration data were expressed in terms of rate (\%) or composition ratio, and the chi-square test and rank-sum test were used for comparison between groups. Logistic regression performed multivariate analysis, and $\mathrm{P}<0.05$ was considered statistically significant.

\section{Results}

There were more female patients $(94.9 \%)$ in the PTC coexistent with the HT group, of which $7.62 \%(9 / 118)$ had accidental cancer, while $6.36 \%(15 / 236)$ of the PTC group had accidental cancer. There were no statistically significant differences between the two groups in age, tumor diameter, the proportion of multiple foci, preoperative serum free thyroxine level, number of lymph node metastasis cases, number of lymph node metastasis in central compartment region, and $\mathrm{T}$ stage $(\mathrm{P}>0.05)$. The female proportions, preoperative serum thyroid-stimulating hormone, antithyroid globulin antibody, and thyroid peroxidase antibody of the PTC coexistent with HT group are all higher than those of the PTC group $(\mathrm{P}<0.05)$. The number of lymph node dissections in the central compartment region of PTC coexistent with the HT group is higher than that in the PTC group $(\mathrm{P}<0.05$; Table 1).

Analysis of the central lymph node metastasis rate in patients with PTC coexistent with HT group showed the male was higher than female $(66.7 \%$ vs. $31.2 \%)$, age $<55$ years was higher than $\geq 55$ years $(36.6 \%$ vs. $20.0 \%)$, tumor diameter $>1 \mathrm{~cm}$ was higher than $\leq 1 \mathrm{~cm}(59.1 \%$ vs. $27.1 \%$ ), and multifocal cancer was higher than single-focal cancer $(51.1 \%$ vs. $21.9 \%)$.

Univariate analysis showed that anti-TGAb positive, tumor diameter $>1 \mathrm{~cm}$, and multifocal cancer were all correlated with central lymph node metastasis $(\mathrm{P}<0.05)$, while TPO-Ab status and gender were not correlated with central compartment lymph node metastasis $(\mathrm{P}>0.05$; Table 2).

Multivariate analysis of central lymph node metastasis in PTC coexistent with the HT group. Univariate analysis was used to introduce statistically significant correlation factors into logistic regression analysis. The results showed that tumor diameter $>1 \mathrm{~cm}$ and multifocal cancer were risk factors for central compartment region lymph node metastasis in the T1/T2 cN0 PTC coexistent with HT group $(\mathrm{P}<0.05$; Table 3).

Analysis of risk factors of central compartment lymph node metastasis in patients with T1/T2 cN0 PTC the clinicopathological data of all the patients with stage T1/ T2 cN0 PTC were introduced into Logistic regression. The results showed that TSH, HT, and thyroid membrane invasion had no statistical difference with lymph node metastasis in the central compartment region $(\mathrm{P}>0.05)$. Male, age $<55$ years old, tumor diameter $>1 \mathrm{~cm}$, and multifocal carcinoma are all risk factors for lymph node metastasis in the central compartment region $(\mathrm{P}<0.05$; Table 4).

\section{Discussion}

The body has many similarities in response to tumor cells and inflammation. Inflammatory cells and cytokines in tumor tissue may play a key role in tumor growth, progression, and immunosuppression. Nearly $15 \%$ of clinical malignancies can be attributed to inflammation. Virchow first proposed in 1863 that chronic inflammation was associated with malignant tumors, and the association has now been showed in various malignant tumors 
Table 1 Comparison of general data of PTC coexistent with HT group and PTC group

\begin{tabular}{|c|c|c|c|c|}
\hline Clinicopathologic features & $\begin{array}{l}\text { PTC coexistent with HT } \\
\text { group }(n=118)\end{array}$ & PTC group $(n=236)$ & $\chi^{2} / Z / t$ & $P$ value \\
\hline Gender, n (\%) & & & 14.735 & $<0.001$ \\
\hline Female & $112(94.9)$ & $187(79.2)$ & & \\
\hline$>1$ & $22(18.6)$ & $48(20.3)$ & & \\
\hline$\leq 1$ & $96(81.4)$ & $188(79.7)$ & & \\
\hline Multifocality, n (\%) & & & 0.220 & 0.639 \\
\hline $\mathrm{TSH}(\bar{x} \pm \mathrm{s}, \mathrm{mlU} / \mathrm{L})$ & $2.84 \pm 1.76$ & $2.43 \pm 1.62$ & 2.221 & 0.027 \\
\hline Anti-TGAb ( $\bar{x} \pm \mathrm{s}, \mathrm{mlU} / \mathrm{L})$ & $177.7 \pm 151.8$ & $24.0 \pm 15.3$ & 10.968 & $<0.001$ \\
\hline TPO-Ab ( $\bar{x} \pm \mathrm{s}, \mathrm{mlU} / \mathrm{L})$ & $522.3 \pm 556.0$ & $38.6 \pm 14.4$ & 9.447 & $<0.001$ \\
\hline Patients with lymph node metastasis, n (\%) & $39(33.1)$ & $88(37.3)$ & 0.614 & 0.433 \\
\hline $\begin{array}{l}\text { Number of lymph nodes dissected in central } \\
\text { compartment area }(\bar{x} \pm \mathrm{s})\end{array}$ & $7.6 \pm 5.2$ & $4.0 \pm 3.4$ & 7.012 & $<0.001$ \\
\hline $\begin{array}{l}\text { Number of lymph node metastases in central } \\
\text { compartment area }(\bar{x} \pm \mathrm{S})\end{array}$ & $0.83 \pm 1.8$ & $0.80 \pm 1.5$ & 0.163 & 0.871 \\
\hline
\end{tabular}

PTC, papillary thyroid carcinoma; HT, Hashimoto's thyroiditis; $\mathrm{FT}_{4}$, free thyroxine; TSH, thyroid stimulating hormone; anti-TGAb, antithyroglobulin antibody; TPO-Ab, thyroid peroxidase antibody.

including cervical cancer, colon cancer, and stomach cancer (13). In 1952, Lindsay et al. (6) studied PTC and $\mathrm{HT}$, and relevant studies are continuing to this day. In the past 30 years, the incidence of PTC and HT has increased rapidly (1-5). Also, the incidence of PTC coexistent with HT has increased significantly. The relationship between the two has become a research focus. Studies have shown the incidence of PTC in HT patients is 1.3-2 times higher than other non-HT thyroid benign diseases (14). Many studies and mate analysis showed that the incidence of PTC combined with HT was $5-85 \%$, which may be related to the race, gender, environment and other factors of the investigated population, also closely related to the differences in specimen collection methods, surgical scope, HT pathological diagnostic criteria and other medical services $(7,8,14-16)$. In this study, PTC coexistent with HT accounted for $33.3 \%$ of the patients with T1/ T2 cN0 PTC was still within the reported range, but significantly higher than the domestic relevant reports. The reason may be related to the geographical environment, ethnic composition, and dietary habits of the Mongolian plateau (17).

The studies of Kwak et al. (18) and Liang et al. (19) confirmed that the TSH of patients with PTC coexistent with $\mathrm{HT}$ was higher than patients with $\mathrm{HT}$ alone, and our results are consistent with them. Research by Liu (14) and Resende de Paiva et al. (15) showed that HT is an independent risk factor for PTC, and believe that the 
Table 2 Univariate analysis of lymph node metastasis in the central region of PTC coexistent with the HT group

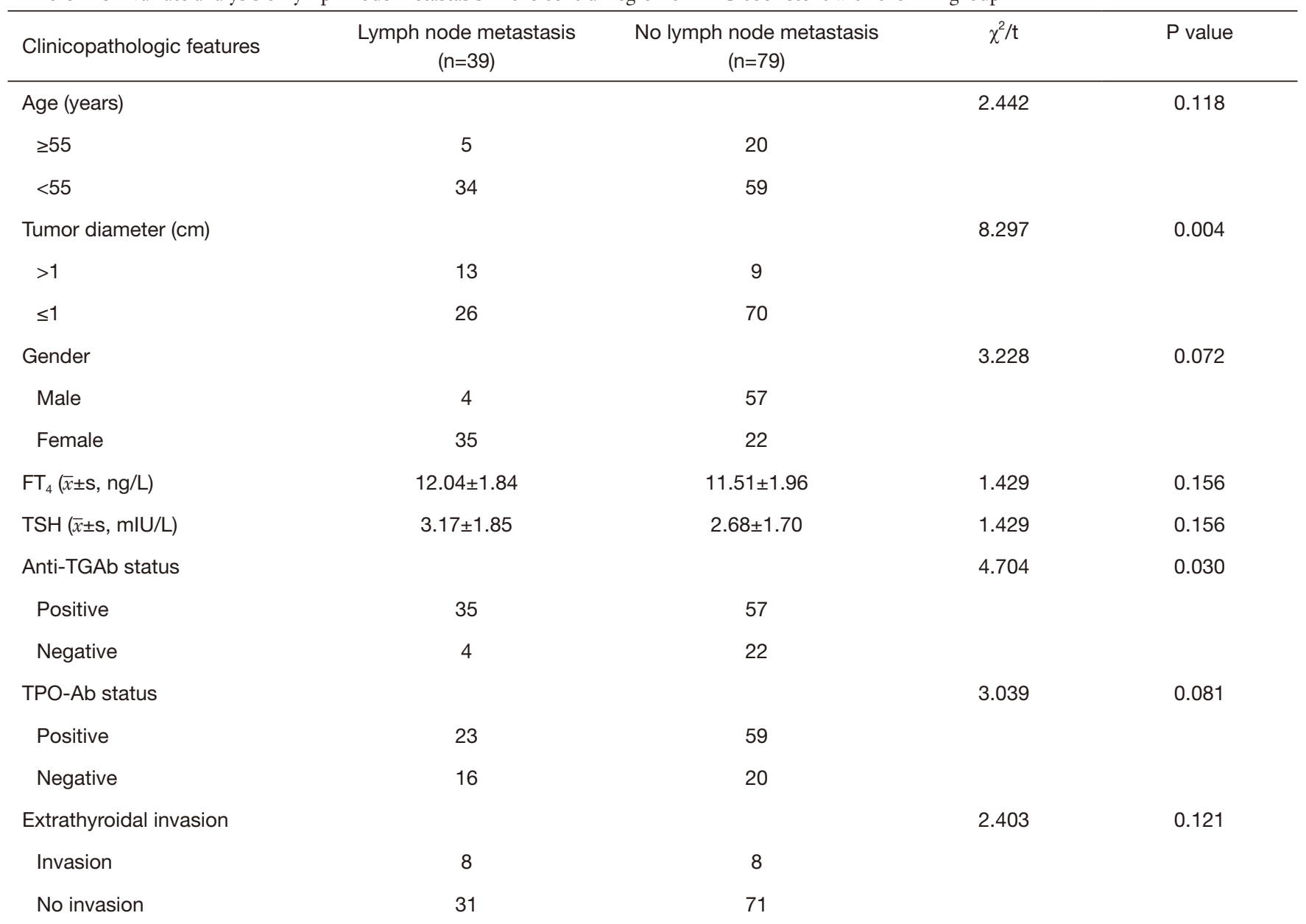

PTC, papillary thyroid carcinoma; HT, Hashimoto's thyroiditis. Anti-TGAb greater than $60.00 \mathrm{IU} / \mathrm{mL}$ is positive; $0.00-60.00 \mathrm{IU} / \mathrm{mL}$ is negative. TPO-Ab greater than $60.00 \mathrm{IU} / \mathrm{mL}$ is positive; $0.00-60.00 \mathrm{IU} / \mathrm{mL}$ is negative.

autoimmune response caused by HT leads to an increase in $\mathrm{TSH}$, which increases the risk of thyroid tumors. Currently, it is believed that thyroid stimulating hormone (TSH) and its homologous receptor (TSHR) are essential for the proliferation and function of thyroid cells. The signal cascade induced by activated TSHR has been shown to be the oncogenic pathway of thyroid cancer, especially in tumors carrying mutations of the B-Raf proto-oncogene V600E. With the increase of TSH levels, the incidence of PTC gradually increases. Studies have found that not only does the incidence of PTC gradually increase with the increase of TSH levels, but the average TSH level of patients with stage III-IV PTC is significantly higher than that of patients with stage I-II PTC. Perhaps TSHR can be used as a candidate target for anticancer therapy (20).

Studies have shown that most patients with PTC coexistent with HT are young women, the proportion of multifocal cancer is higher, the tumor size is smaller, and the central lymph node metastasis rate and $\mathrm{T}$ stage are lower $(14,15,21,22)$. Our results showed that the proportion of female patients with PTC coexistent with HT was higher. However, there was no statistically significant difference in the lymph node metastasis rate in the central compartment region between the PTC coexistent with the HT group and the PTC group alone $(\mathrm{P}>0.05)$, which may be related to the fact that the subjects in this study were only at this stage T1/T2 cN0 PTC and mainly had thyroid microcarcinoma. Current studies have shown that high levels of estrogen and reproductive factors may be responsible for gender differences in PTC (23). The incidence of PTC is the highest in premenopausal women, suggesting that female sex hormones may promote the occurrence and progression 
Table 3 Logistic regression analysis of risk factors for T1/T2 cN0 PTC coexistent with HT group lymph node metastasis in central compartment region

\begin{tabular}{|c|c|c|c|c|c|c|}
\hline Variables & B & SE. & Wald $\chi^{2}$ & $P$ value & OR & $95 \% \mathrm{Cl}$ \\
\hline Multifocal carcinoma (yes vs. no) & 1.140 & 0.428 & 7.091 & 0.008 & 3.128 & $1.351-7.241$ \\
\hline Anti-TGAb (positive vs. negative) & 1.015 & 0.621 & 2.677 & 0.102 & 2.760 & $0.818-9.314$ \\
\hline Constant & -2.281 & 0.621 & 13.898 & 0.000 & 0.102 & - \\
\hline
\end{tabular}

-, no data. PTC, papillary thyroid carcinoma.

Table 4 Logistic regression analysis of influencing factors of central compartment lymph node metastasis in patients with cN0 PTC in stage T1/ $\mathrm{T} 2$

\begin{tabular}{|c|c|c|c|c|c|c|c|}
\hline \multirow{2}{*}{ Variables } & \multirow{2}{*}{$\mathrm{B}$} & \multirow{2}{*}{ SE. } & \multirow{2}{*}{ Wald $\chi^{2}$} & \multirow{2}{*}{$P$ value } & \multirow{2}{*}{ OR } & \multicolumn{2}{|c|}{$95 \% \mathrm{Cl}$} \\
\hline & & & & & & Lower & Upper \\
\hline Gender (male vs. female) & 0.858 & 0.324 & 7.002 & 0.008 & 2.358 & 1.249 & 4.451 \\
\hline Age (<55 vs. $\geq 55)$ & -0.822 & 0.307 & 7.002 & 0.008 & 0.440 & 0.241 & 0.803 \\
\hline Tumor diameter (>1 vs. $\leq 1 \mathrm{~cm})$ & 1.011 & 0.289 & 12.235 & 0.000 & 2.749 & 1.560 & 4.845 \\
\hline Thyroid capsule invasion (yes vs. no) & -0.222 & 0.321 & 0.477 & 0.490 & 0.801 & 0.241 & 0.803 \\
\hline TSH (<2.5 vs. $\geq 2.5$ ulU/mL) & 0.402 & 0.245 & 2.684 & 0.101 & 1.495 & 0.924 & 2.418 \\
\hline HT (yes vs. no) & -0.146 & 0.261 & 0.314 & 0.575 & 0.864 & 0.518 & 1.441 \\
\hline Constant & -1.194 & 0.231 & 26.818 & 0.000 & 0.303 & - & - \\
\hline
\end{tabular}

PTC, papillary thyroid carcinoma; HT, Hashimoto's thyroiditis; -, no data.

of thyroid cancer. In vitro and animal studies have found that sex hormones can affect the proliferation of thyroid cells, of which estrogen and estrogen receptors are currently the most studied. Estrogen and estrogen receptors are expected to become prognostic indicators and potential therapeutic targets for the survival of female patients $(23,24)$. It is not clear whether HT influences the lymph nodes in the central compartment region of cN0 PTC in the T1/ $\mathrm{T} 2$ stage. There are few relevant studies and insufficient significant data support.

Patients with PTC coexistent with HT often have more lymph nodes in the neck, and preoperative color Doppler ultrasound was challenging determining lymph node metastasis. In this study, there was no significant difference in the number of central compartment lymph node metastases between the PTC coexistent with the HT group and the PTC group. The research by Kwak et al. (18) showed that there was no significant difference in the number of central compartment lymph node metastases between the PTC coexistent with the HT group and the PTC group ( $\mathrm{P}=0.692)$. PTC combined with HT causes diffuse hyperplasia of the thyroid gland, dense adhesion between the glands and surrounding tissues, front neck VI area, multiple enlarged lymph nodes, which increases the difficulty of preserving the parathyroid glands in the initial position during the operation. This study showed that $17 \%$ (20/118) of patients in the PTC coexistent with HT group had over ten central compartment lymph nodes, but none of them had no lymph node metastasis. It suggests the number of central lymph nodes in patients with PTC and HT is increasing, and it is difficult to judge the metastasis by the naked eye during the operation. Therefore, clarifying the risk factors of central lymph node metastasis is beneficial to clarify the necessity of lymph node dissection and the choice of dissection scope. The research of Liang et al. (19) showed that age $<45$ years and multifocal cancer are risk factors for lymph node metastasis in patients with PTC coexistent with HT. The study by Zhu et al. (22) showed 
that age $<45$ years, tumor diameter $>1 \mathrm{~cm}$, and multifocal cancer are risk factors for central compartment lymph node metastasis in patients with PTC coexistent with HT. The multivariate analysis of our study showed that age $<45$ years, tumor diameter $>1 \mathrm{~cm}$, and multifocal carcinoma are risk factors for central lymph node metastasis in patients with T1/T2 cN0 PTC coexistent with HT, which are consistent with the above research conclusions.

Multivariate analysis of other studies showed that HT was a protective factor for lymph node metastasis in the central compartment region of PTC $(21,22)$. The studies of Liu et al. (14) and Zeng et al. (17) showed that HT is not a relevant factor for lymph node metastasis in the central compartment region of PTC. The reasons for the potential differences in the above studies may be related to the pathological and the included subjects. Studies have shown that HT was considered a protective factor for lymph node metastasis in the central compartment region, and the proportions of T3/T4 PTC and nonCN0 PTC were large. However, in recent years, with the highest incidence of T1/T2 stage, cN0 PTC did not find the above situation, it still is to be further research. The research of Song et al. (8) and Liang et al. (19) showed the disease-free survival in patients with PTC coexistent with HT was longer than that in patients with PTC alone. The research of Song et al. (8) and Liang et al. (19) showed the disease-free survival in patients with PTC coexistent with HT was longer than that in patients with PTC alone. The study of Kwak et al. (18) showed that HT was a protective factor for PTC recurrence; tumor diameter $>1 \mathrm{~cm}$, central compartment lymph node metastasis ratio, and BRAF V600 mutation were all risk factors for PTC recurrence. The median follow-up period of the above studies was 27 to 96 months, which still needs to be confirmed by many longterm prospective studies. Therefore for PTC coexistent HT patients, diagnosis and treatment should be conducted according to the existing PTC diagnosis and treatment principles.

The American Thyroid Association guidelines (2015 edition) recommend that non-invasive T1/T2 cN0 PTC does not require PCCND, and the 5-year survival rate of patients with active monitoring and active intervention is $98.2 \%$ (10). Park et al. (25) conducted a questionnaire survey among patients with thyroid cancer after surgery and showed that $69.2 \%$ of patients with thyroid cancer approved of the operation with a low recurrence rate and could accept a slight amount of increased risk of complications. Chen et al. (26) conducted a retrospective study of 18,376
PTC patients and showed PCCND could better remove potential lymph node metastasis. Further, it can significantly reduce the local recurrence rate of cN0 PTC, beneficial for monitoring postoperative recurrence, metastasis, and the effect of postoperative radioiodine treatment with the change of thyroglobulin. However, the increase in the risk of temporary recurrent laryngeal nerve injury and permanent hypocalcemia. Therefore, a comprehensive analysis of various risk factors should be conducted before surgery and consultation with patients, then determine the treatment plan. Therefore, in the adequate protection of parathyroid and nerve integrity, the primary surgery to give PCCND can reduce the risk of recurrence and metastasis.

There are still shortcomings in this study: (I) the confounding factors in this study (including not distinguishing aggressive PTC subtypes, not comparing differences among ethnic groups) may impact the statistical results; (II) the involved papillary thyroid carcinoma samples need to meet the conditions of cN0, T1/T2 Stage, and Hashimoto's thyroiditis at the same time. Moreover, this study is a single-center study, so the sample size is small; (III) there are not enough statistical data and tracking data for postoperative complications. Therefore, multicenter collaboration and long-term follow-up are needed to obtain more reliable results in the future.

In summary, PTC combined with HT is more common in women, and TSH level in patients with PTC coexistent with HT is higher than that in patients with PTC alone. $\mathrm{HT}$ is not a related factor of lymph node metastasis in the central compartment region of cN0 PTC in the T1/T2 stage. Regardless of whether it is combined with HT, tumor diameter $>1 \mathrm{~cm}$ and multifocal cancer are risk factors for central compartment lymph node metastasis in patients with T1/T2 cN0 PTC. PCCND should be actively performed during surgery.

\section{Acknowledgments}

Funding: None.

\section{Footnote}

Reporting Checklist: The authors have completed the STROBE reporting checklist. Available at http://dx.doi. org/10.21037/gs-20-699

Data Sharing Statement: Available at http://dx.doi. org/10.21037/gs-20-699 
Conflicts of Interest: All authors have completed the ICMJE uniform disclosure form (available at http://dx.doi. org/10.21037/gs-20-699). The authors have no conflicts of interest to declare.

Ethical Statement: The authors are accountable for all aspects of the work in ensuring that questions related to the accuracy or integrity of any part of the work are appropriately investigated and resolved. The study was reviewed and approved by the Ethics Committee of Clinical Investigation in the Inner Mongolia People's hospital. All procedures performed in this study involving human participants were in accordance with the Declaration of Helsinki (as revised in 2013). Individual consent for this retrospective analysis was waived.

Open Access Statement: This is an Open Access article distributed in accordance with the Creative Commons Attribution-NonCommercial-NoDerivs 4.0 International License (CC BY-NC-ND 4.0), which permits the noncommercial replication and distribution of the article with the strict proviso that no changes or edits are made and the original work is properly cited (including links to both the formal publication through the relevant DOI and the license). See: https://creativecommons.org/licenses/by-nc-nd/4.0/.

\section{References}

1. Mao Y, Xing M. Recent incidences and differential trends of thyroid cancer in the USA. Endocr Relat Cancer 2016;23:313-22.

2. Siegel RL, Miller KD, Jemal A. Cancer statistics, 2015. CA Cancer J Clin 2015;65:5-29.

3. Chai L, Han D, Li J, Lv Z. The construction and analysis of gene co-expression network of differentially expressed genes identifies potential biomarkers in thyroid cancer. Transl Cancer Res 2018;7:1235-43.

4. Bothra N, Shah N, Goroshi M, et al. Hashimoto's thyroiditis: relative recurrence risk ratio and implications for screening of first-degree relatives. Clin Endocrinol (Oxf) 2017;87:201-6.

5. Ahmed R, Al-Shaikh S, Akhtar M. Hashimoto thyroiditis: a century later. Adv Anat Pathol 2012;19:181-6.

6. Lindsay S, Dailey ME, Friedlanger J, et al. Chronic thyroiditis: A clinical and pathologic study of 354 patients. J Clin Endocrinol Metab 1952;12:1578-600.

7. Dobrinja C, Makovac P, Pastoricchio M, et al. Coexistence of chronic lymphocytic thyroiditis and papillary thyroid carcinoma. Impact on presentation, management, and outcome. Int J Surg 2016;28 Suppl 1:S70-74.

8. Song E, Jeon MJ, Park S, et al. Influence of coexistent Hashimoto's thyroiditis on the extent of cervical lymph node dissection and prognosis in papillary thyroid carcinoma. Clin Endocrinol (Oxf) 2018;88:123-8.

9. Park JH, Lee YS, Kim BW, et al. Skip lateral neck node metastases in papillary thyroid carcinoma. World J Surg 2012;36:743-7.

10. Haugen BR, Alexander EK, Bible KC, et al. 2015 American Thyroid Association Management Guidelines for Adult Patients with Thyroid Nodules and Differentiated Thyroid Cancer: The American Thyroid Association Guidelines Task Force on Thyroid Nodules and Differentiated Thyroid Cancer. Thyroid 2016;26:1-133.

11. Perros P, Boelaert K, Colley S, et al. Guidelines for the management of thyroid cancer. Clin Endocrinol (Oxf) 2014;81 Suppl 1:1-122.

12. Amin MB, Edge SB, Greene FL, et al. AJCC Cancer Staging Manual Eighth Edition. Switzerland: Springer International Publishing, 2017:873-90.

13. Balkwill F, Mantovani A. Inflammation and cancer: Back to Virchow? Lancet 2001;357:539-45.

14. Liu X, Zhu L, Cui D, et al. Coexistence of histologically confirmed Hashimoto's thyroiditis with different stages of papillary thyroid carcinoma in a consecutive Chinese cohort. Int J Endocrinol 2014;2014:769294.

15. Resende de Paiva C, Grønhøj C, Feldt-Rasmussen U, et al. Association between Hashimoto's thyroiditis and thyroid cancer in 64,628 patients. Front Oncol 2017;7:53.

16. Lee JH, Kim Y, Choi JW, et al. The association between papillary thyroid carcinoma and histologically proven Hashimoto's thyroiditis: A meta-analysis. Eur J Endocrinol 2013;168:343-9.

17. Zeng R, Shou T, Yang KX, et al. Papillary thyroid carcinoma risk factors in the Yunnan plateau of southwestern China. Ther Clin Risk Manag 2016;12:1065-74.

18. Kwak HY, Chae BJ, Eom YH, et al. Does papillary thyroid carcinoma have a better prognosis with or without Hashimoto thyroiditis? Int J Clin Oncol 2015;20:463-73.

19. Liang J, Zeng W, Fang F, et al. Clinical analysis of Hashimoto thyroiditis coexistent with papillary thyroid cancer in 1392 patients. Acta Otorhinolaryngol Ital 2017;37:393-400.

20. Rowe CW, Paul JW, Gedye C, et al. Targeting the 
TSH receptor in thyroid cancer. Endocr Relat Cancer 2017;24:R191-R202.

21. Wen X, Wang B, Jin Q, et al. Thyroid Antibody Status is Associated with Central Lymph Node Metastases in Papillary Thyroid Carcinoma Patients with Hashimoto's Thyroiditis. Ann Surg Oncol 2019;26:1751-8.

22. Zhu F, Shen YB, Li FQ, et al. The effects of Hashimoto Thyroiditis on Lymph node metastases in unifocal and multifocal papillary thyroid carcinoma: a retrospective Chinese cohort study. Medicine (Baltimore) 2016;95:e2674.

23. Moleti M, Sturniolo G, Di Mauro M, et al. Female Reproductive Factors and Differentiated Thyroid Cancer. Front Endocrinol (Lausanne) 2017;8:111.

24. Yi JW, Kim SJ, Kim JK, et al. Upregulation of the ESR1

Cite this article as: Jin $\mathrm{K}, \mathrm{Li} \mathrm{L}$, Liu Y, Wang X. The characteristics and risk factors of central compartment lymph node metastasis in $\mathrm{cN} 0$ papillary thyroid carcinoma coexistent with Hashimoto's thyroiditis. Gland Surg 2020;9(6):2026-2034. doi: $10.21037 / g s-20-699$
Gene and ESR Ratio (ESR1/ESR2) is Associated with a Worse Prognosis in Papillary Thyroid Carcinoma: The Impact of the Estrogen Receptor $\alpha / \beta$ Expression on Clinical Outcomes in Papillary Thyroid Carcinoma Patients. Ann Surg Oncol 2017;24:3754-62.

25. Park JW, Yoo JS, Yun JK, et al. An online questionnaire survey on preferred timing for the diagnosis and management of thyroid carcinoma in general population in Korea. Ann Surg Treat Res 2016;90:297-302.

26. Chen L, Wu YH, Lee CH, et al. Prophylactic central neck dissection for papillary thyroid carcinoma with clinically uninvolved central neck lymph nodes: a systematic review and meta-analysis. World J Surg 2018;42:2846-57.

(English Language Editor: J. Chapnick) 